\title{
NGO Support Software Solution: for effective reachability
}

\author{
Janhavi Desale $^{\mathrm{a}}$, Kunal Gautam ${ }^{\mathrm{a}}$, Saish Khandare ${ }^{\mathrm{a}}$, Vedant Parikh ${ }^{\mathrm{a}}$, Dhanashree Toradmalle ${ }^{\mathrm{a}}$ \\ Shah \& Anchor Kutchhi Engineering College, Mumbai-400088, India \\ E-mail: kunal77gautam@gmail.com
}

Received:24 June 2020; Accepted: 15 July 2020; Published: 08 December 2020

\begin{abstract}
In India there are currently a lot of NGOs working for noble causes. Citizens are also eager to contribute. Unfortunately for a lot of these NGOs there is a shortage or absence of IT infrastructure, hindering their reach and effectiveness. We aim to aid such NGOs and provide them with the necessary IT infrastructure to optimize use of resources and increase their reach for food and money donations. The project includes a cross platform mobile application which will help them manage their volunteers, get orders by spreading awareness through a social media module to connect to people who wish to support them in this noble cause. There are many freelancing developers and existing apps, the goal is to extract all the best features, figure out best platforms, harness latest trends and develop the app at a cost that the NGO can afford. Our literature survey of existing app gave insights about designing the necessary modules that we must have. To have the analysis of people's attitude, habits and trends, studying papers related to social media trends inspired us to harness its power. The study of other kinds of systems using mobile applications, encouraged us to consider options of $100 \%$ cost free background, enabling us to generate an economical solution.
\end{abstract}

Index Terms: NGO support software, volunteer management system, donation management system.

\section{Introduction}

Today numerous NGOs are dynamic in India for respectable causes. Residents also are enthused to give a lot of effort. The issue is the absence of a stage for these NGOs to contribute their contemplations expansion to ill-advised administration and nonattendance of specialized IT support. Our project targets helping such NGOs as far as IT support for expanding their social reach and volunteer administration.

In a nation where a huge number of individuals go hungry consistently, a NGO thought of an activity to bolster the poor. The NGO, gathers leftovers from eateries, clubs and gatherings in Mumbai, stores it in vans and conveys it to the poor before the nourishment gets stale. The NGO for the most part gathers extra nourishment staples like rotis (chapatti or wheat-made level bread), rice, vegetables and dal to be dispersed.

As a feature of the activity, multiple GPRS-prepared vans make rounds of boulevards close to clinics and financially deprived areas to give nourishment to the destitute individuals. The NGO has a devoted helpline where a person can contact and our committed vans get the excess meals and redistribute it instantly, along these lines guaranteeing that the nature of the nourishment stays flawless. It is proposed to make a versatile application for the donors, and the food donation module as to facilitate the chain of the Food to the Needy inside limited capacity to focus time with the assistance of volunteers.

As of now they are working with no utilization of innovation in their chain of dissemination. In this manner, we propose to embrace this undertaking to help such NGOs increment its scope. The task comprises of two applications client application and administrator application.

The essential customer prerequisite is to make a framework that authorizes the attention of that NGO among the network, enables residents to effectively take part and enable the power to deal with the volunteers for the occasions headed by the NGO, utilizing IT stages that are affordable as well as productive. The Users have the option to enlist for volunteering and furthermore share their media via web-based networking media. The administrator has the option to view enrolled volunteer dashboard. The above discussed application ought to be accessible on both Android and iOS platform. In this manner a Cross Platform Application needs to be developed.

The objective of our research is based on finding the solution to the current mobile ecosystem involving factors ranging from NGO Adaptability, Software Development Constraints, Public Attitude. These are considered as challenges to come up with an economical solution that could present a best viable solution and can provide means for developers to easily develop applications with minimal labour and maximum platform outreach, economical costing platform affordable for NGO. It enables the NGO to run and maintain the app, and yet be user friendly, and provide optimal user experience that 
will help the NGO to reach out and get better public support. The goal is to achieve the most viable product considering above discussed factors.

\section{Review of Prior Work}

\subsection{Comparative analysis of prior work :}

Table 1. Comparative Analysis of existing Support Applications

\begin{tabular}{|c|c|c|c|c|c|}
\hline Name & Donate Food & Donate Money & $\begin{array}{l}\text { Register for } \\
\text { volunteering }\end{array}$ & Order free food & Social Media \\
\hline Aahar & $\checkmark$ & $x$ & $x$ & $x$ & $x$ \\
\hline Roti Bank - & $\checkmark$ & $\checkmark$ & $\checkmark$ & $x$ & $x$ \\
\hline \multicolumn{6}{|l|}{ Haryana } \\
\hline AIRTBT & $x$ & $x$ & $\checkmark$ & $\checkmark$ & $x$ \\
\hline Bhoojan & $\checkmark$ & $x$ & $x$ & $x$ & $x$ \\
\hline ShareTheMeal & $\checkmark$ & $x$ & $x$ & $x$ & $\checkmark$ \\
\hline Food4needy & $\checkmark$ & $\checkmark$ & $x$ & $x$ & $x$ \\
\hline
\end{tabular}

\subsection{Remarks :}

All of the apps missed integration of modules of volunteers along with social media except for one that was for a worldwide program and not a local NGO. Therefore on the basis of understanding the prior work in this field and the current requirement of our proposed system and the scenario in today's world, there is a pressing need to have a volunteer management system along with social awareness module that will bridge the gap between people who have taken the initiative and those who simply want to contribute their share of effort.

\section{Requirement and feasibility analysis :}

\subsection{Database comparison :}

Table 2. Comparison of database alternatives

\begin{tabular}{lll}
\hline Basis of comparison & Firebase & MS SQL Server \\
\hline Data Model & Stored as JSON Tree & Table \\
DB Engine ranking & Score- 2.79 Rank- 79 Overall, 13 Key- Value & Score- 94 Rank- 3 Overall, 3 RDBMS \\
& Pairs & \\
Website & Firebase.google.com & Microsoft.com./enus/SQL-server \\
Developer & Google & Microsoft \\
Database Schema & Schema Free & Yes \\
SQL & No & Yes \\
Supported language & Java, JavaScript, Swift & C++, Delphi, Go Java, JavaScript, PHP, \\
& & Python, Ruby, Visual Basic \\
\hline
\end{tabular}

We opted for Google Firebase as our backend support as it has got great synergy with Android Studio which is also developed by Google and facilitates seamless integration. We also cut down on our server setup costs that would be imposed if we were to use MS SQL Server or any other hosted server for that matter. 


\subsection{Feasibility study( Firebase costing) :[2]}

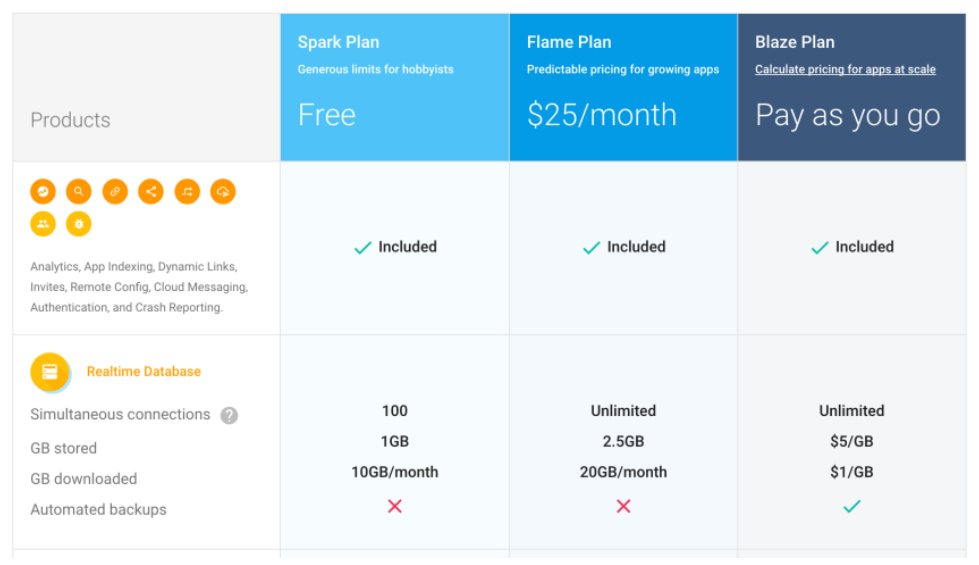

Fig.1. Available Firebase plans

In The above contrast Firebase seemed to be a better option as the Spark plan is free and is also well documented. As it provides sufficient services which will be required by the applications that are for storing real time data Firebase Realtime can be used and for storing images and posts Firebase Storage can be used and be integrated efficiently.

\subsection{Platform comparison :[3,4]}

Table 3. Comparison of native and cross platform applications

\begin{tabular}{ccc}
\hline Comparison Criteria & Mobile & Website \\
\hline Cost & High & Low \\
Time & Long & Short \\
Maintenance & Complex & Easy \\
Expertise & Java, Dart, XML & PHP, JavaScript, HTML \\
UI & Excellent & Good \\
Functionality & Easy & Complex \\
\hline
\end{tabular}

Users would not want to wait while the page fetches the required data again and again in case of a website, instead a mobile application would be a better option because of better UI and functionality. We feel for this project a mobile application will be a better bid.

\subsection{Feasibility study(Cross platform):[1,2]}

Table 4. Comparison of native and cross platform applications

\begin{tabular}{lll}
\hline Comparison Criterion & Cross Platform & Native \\
\hline Quality of UX & Excellent & Not as good as Cross Platform \\
Apps Quality & High & Medium \\
Potential Users & Large- multiple platforms & Constrained to a specific platform \\
App development cost & Low & High \\
\hline
\end{tabular}

Comparing cross platform applications Flutter had the following advantages:

- $\quad$ Provides Better UI

- $\quad$ Better Learning \& Productive Curve

- Fewer Dependencies on features

- $\quad$ Better Documentation and tutorials

To maximize reach both iOS and Android would be needed and thus better to use a cross platform option. From the available cross platforms such as React, Ionic and Flutter. We chose Flutter due to the above mentioned features. Our Project will contain two modules, one for the User and one for the Admin. 


\subsection{Payment Gateway}

We choose RazorPay since it gives access to all payment modes including credit card, debit card, net banking, UPI and almost all popular wallets including Google Pay, JioMoney, Mobikwik, PayUmoney, Airtel Money, FreeCharge, Ola Money and PayZapp.

\subsection{Software Requirements of the research}

Flutter, Android Studio, 4GB RAM, cell phone with internet connectivity.

\section{Software Development process:}

The software development process begins with discussing the basic layout of the applications and its functionality details. A rough idea of all the requirements is taken into consideration by the developers after listening to the NGO representatives. First phase begins by trying to incorporate the client's basic needs into different applications which differ due to their designs. Once a client choses a design, a prototype of this design is formulated into a workable application that has all the requirements covered with the desired interface. This prototype is given to the NGO for testing and they come up with more ideas that need to be integrated in the application. This process takes place a few more times till the client is satisfied with the product. Finally, the product is launched on Playstore and Appstore to be used by the end users.

\section{Module-wise functionality}

The NGO Support Software Solution is a set of two applications, the user app and the admin app. The User App will comprise of four modules aimed at providing a donation management and a volunteer management system to the NGO. The Admin App consists of powerful functionalities to manage functions like post approval, volunteer reviewing and updating statistics.

\section{USER APP}

The modules of the user app are:

\subsection{Donate Food Module}

The Donate Food module is the prime functionality of the app. The module has a button called 'Call Helpline' which opens the dial pad with the number of the NGO already populated. The user has access to the numerical statistics of the meals served and the hours of volunteering. The module also has a 'Donation Map' which shows the graphical representation of the NGO’s food donation hotspots on the map of Mumbai.

Working:

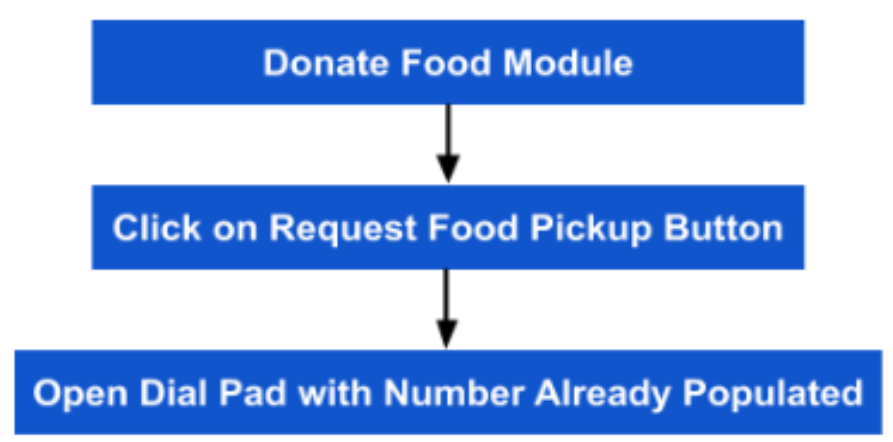

Fig.2. Donate Food module flowchart 


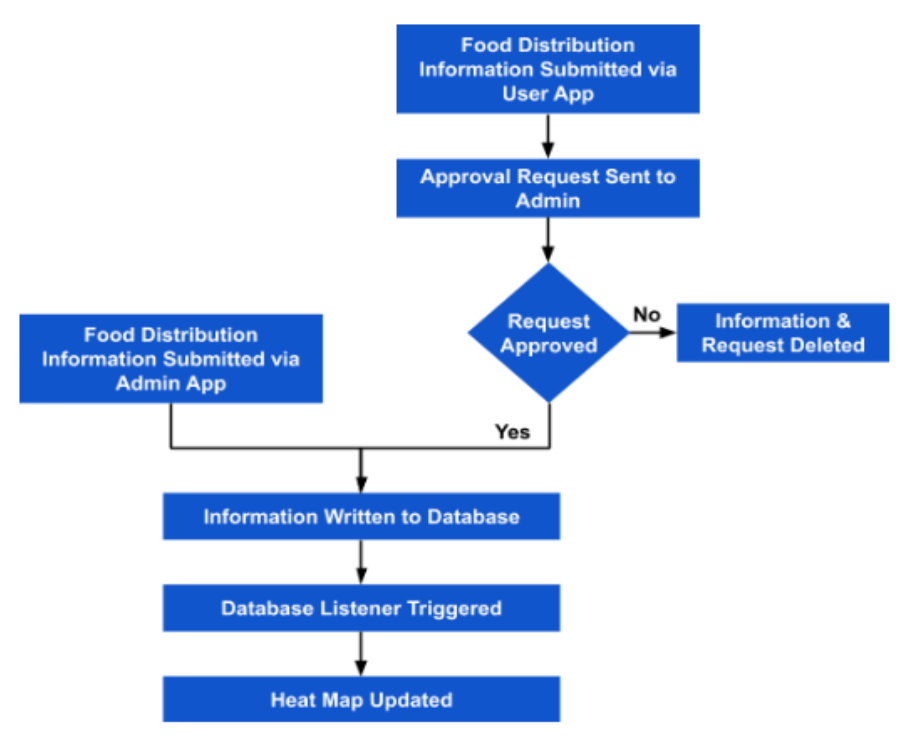

Fig.3. Heat map functionality flowchart

\subsection{Donate Money Module}

NGOs need tremendous backing and funding to function smoothly. This module provides that voluntary support to the NGO in terms of funding. The module has a payment gateway integrated which enables the user to donate money via digital transfers, cards or wallets. The payments are captured by creating and implementing server side logic. The payment details of each transaction are saved to Firebase and the database listener is triggered when new payment is added which sends an email to the donor to acknowledge the payment and confirm the donation.

Working:

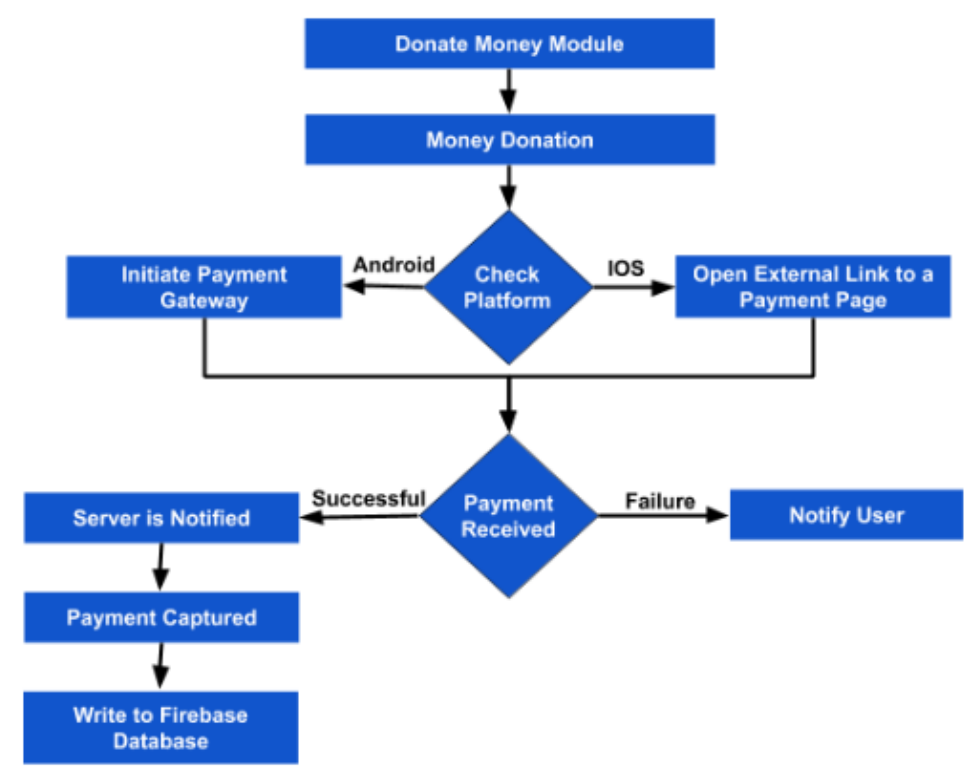

Fig.4. Donate Money module flowchart 


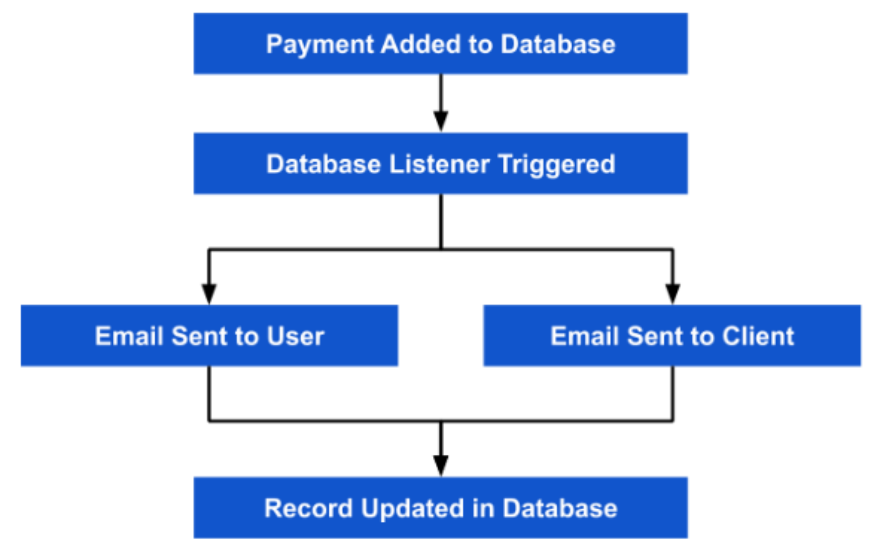

Fig.5. E-mail service flowchart

\subsection{Volunteer Module}

The backbone of any NGO is the taskforce behind the events who ensure the smooth functioning of the events. This module is a vital module when it comes to connecting such likeminded people to the NGO. The volunteer module is where the user can register as a volunteer for the NGO. A personal detail form with a few volunteering preferences are to be filled to register as a volunteer. There is a 'Terms and Condition' checkbox to convey a few statements to the users from the NGO's end. The user is also provided with an opportunity to join the official WhatsApp group of the NGO to be up-to-date with the NGO events and functioning.

Working:

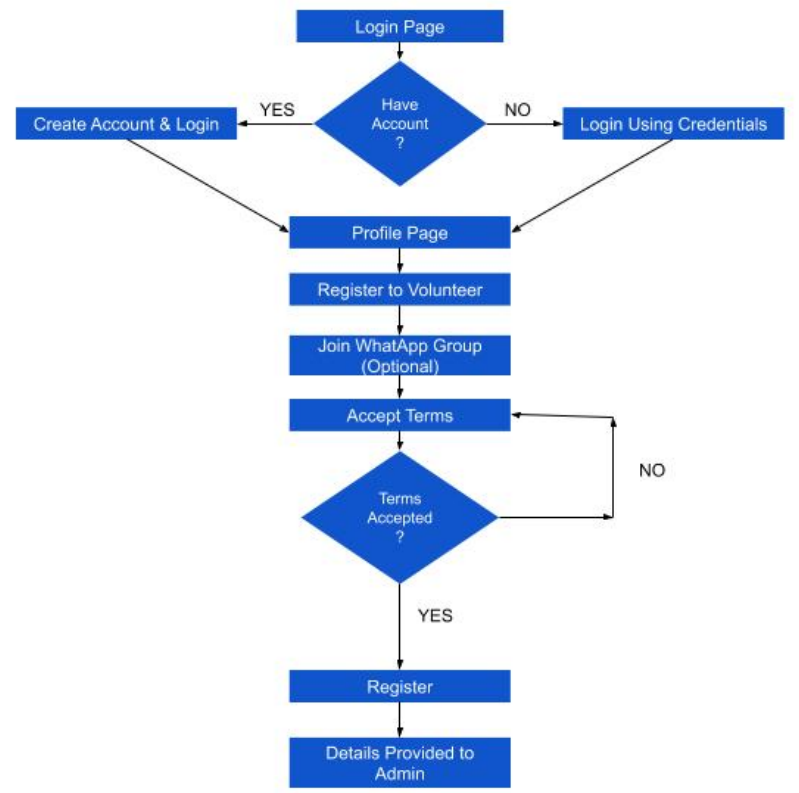

Fig.6. Volunteer Module flowchart

\subsection{Social Module}

The most critical benefit of having an IT infrastructure for a dedicated NGO is to maximize its reach. The Social module is this stepping stone for such NGOs to expand their reach and connect with the masses. In this module the user can view posts related to the NGO events posted by other users and which are verified by the admin through the Admin application. The user can share images and donation details from some event or donation drive with a few details and apply to post it on the Social module. These posts go to the admin for verification and then after approval are displayed on the user application. These posts can be shared on other social media platforms as well which amplifies the reach and connect 
of the NGO.

Working:

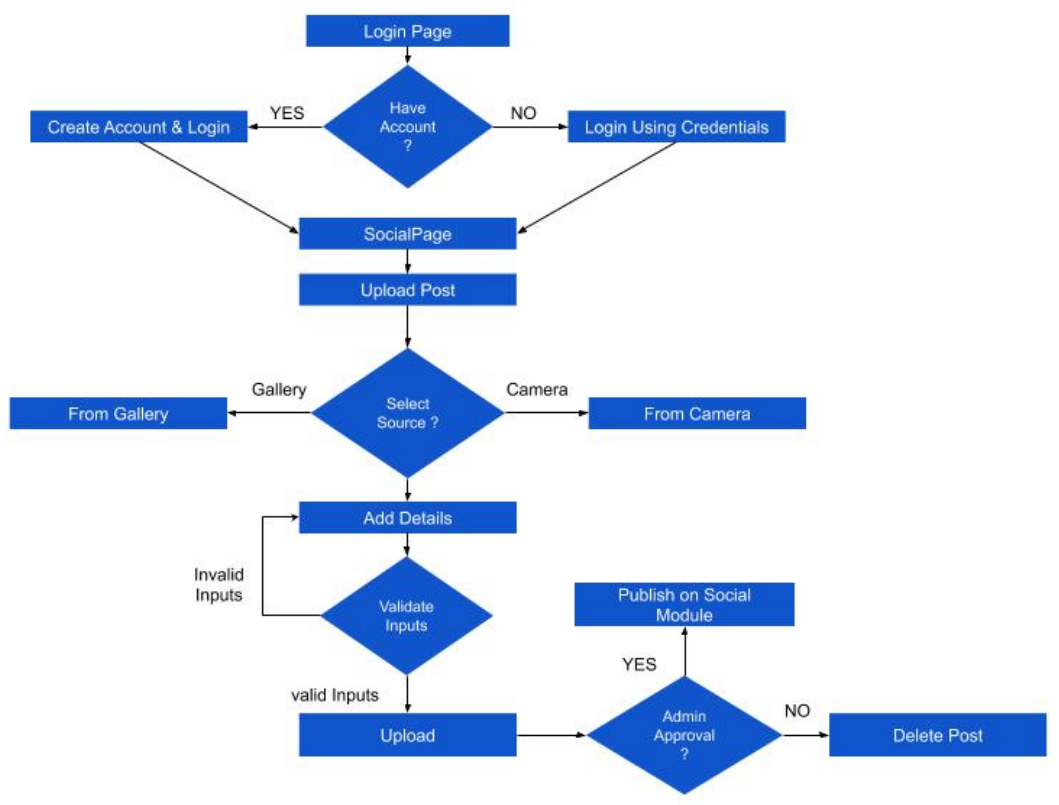

Fig.7. Social Module flowchart

\subsection{About Module}

The About page is where the NGO can link their social media handlers for the users to stay updated with the daily functioning. The page also has a static gallery where posts can be uploaded from the Admin app and is displayed in the user app. The page also has details of the developing team behind this software solution. It has a few lines about the NGO and about our college, Shah \& Anchor Kutchhi Engineering College.

\section{$\underline{\text { ADMIN APP }}$}

The Admin app access is given to only a few trusted individuals from the NGO. The admin app is linked to the user app's Firebase console where data is uploaded. The admin can perform several powerful tasks using this app. The tasks include: accepting volunteer registrations, deciding and give approval as to which posts to be displayed on the user app, upload posts into the static gallery in the 'About page' of the user app, updating statistics which are displayed on the 'Donate Food' module, etc.

\section{Application Screenshots}




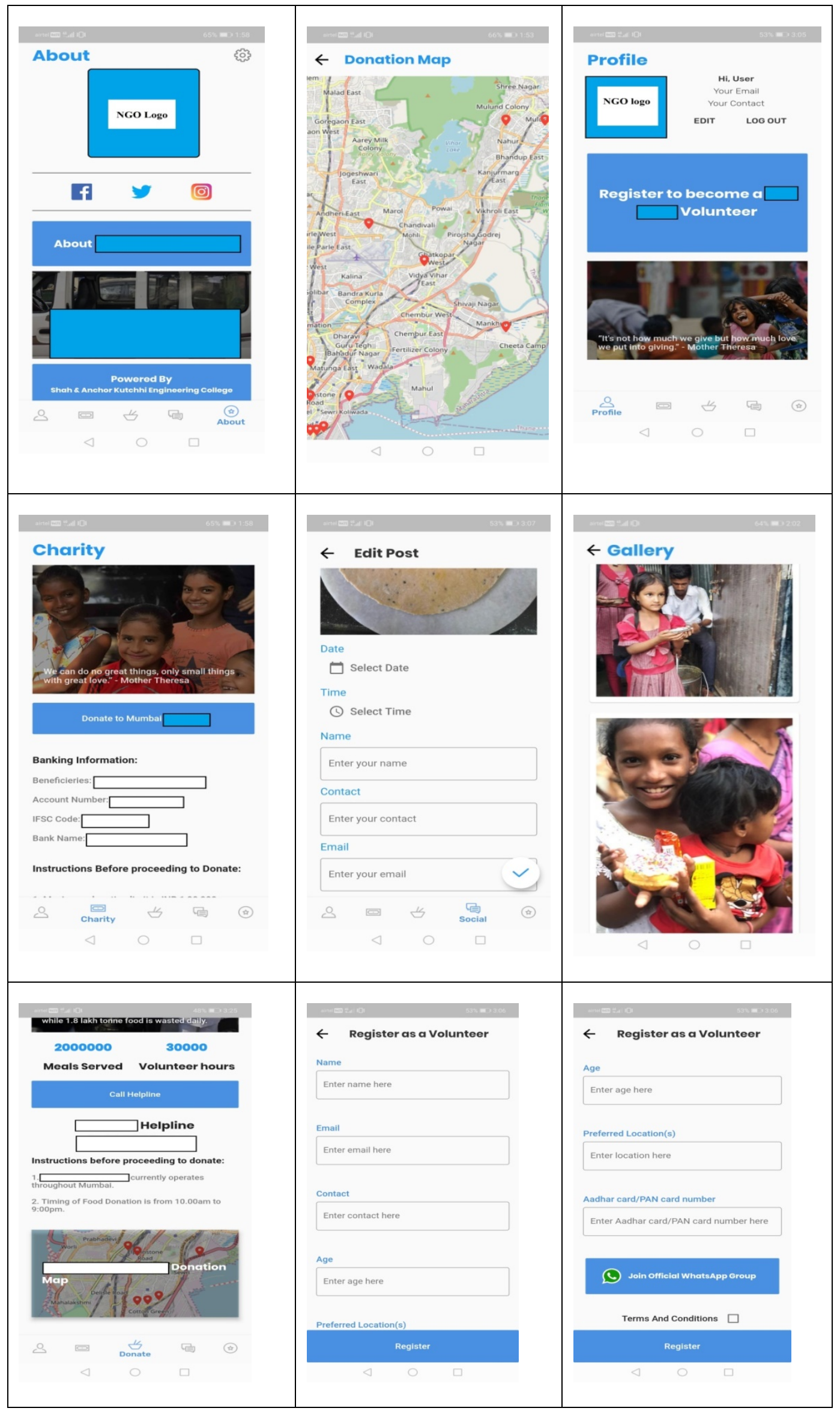

Fig.8. Application Screenshots 


\section{Comprehensive Testing}

The developed app also went through extensive testing. Before any release multiple rounds of alpha and beta testing were conducted. Alpha and beta testing were always conducted with a minimum sample size of 5 and 15 respectively. This enabled any functionality issues/bugs to be caught quickly.

Additionally, the system holds a lot of confidential information of both the users and the NGO. Thus periodically, security audits of the system were conducted. These security audits help safeguard against such data breaches. Additionally each release of the system was put through performance testing before deployment. Factors like memory consumption, CPU usage, battery consumption and app start up times were checked. Furthermore, the app was tested on multiple devices with different hardware and software configurations, i.e. different screen size, operating system, software version, manufacturer, CPU, ram, etc. Only after performance was within acceptable standards on all the different hardware and software configurations was a release launched.

\section{Conclusion:}

It is seen that there are many good small sized NGOs who are working very actively on the ground and do a lot of good work. Unfortunately it is seen that the majority of such NGOs have a lot of trouble expanding and growing their reach. Further, some such NGOs even are forced to shut down after a few years. The three fundamental areas where many such NGOs have problems are: Awareness, infrastructure and management. Our system aids NGOs in these three critical aspects. Furthermore, it is observed that when these three critical aspects are tackled it inevitably also leads to increase in the NGOs funding.

\section{Acknowledgements}

We wish to express our profound gratitude to our Principal Dr. Bhavesh Patel, Research Cell Head Dr.Nilakshi Jain and project guide Ms.Dhanashree Toradmalle for allowing us to go ahead with this project and provide us with this opportunity to explore this domain. We would also like to thank our I/c Head of Department Ms. Swati Nadkarni for her constant encouragement and support towards achieving this goal. We would also like to thank the Review Committee for their invaluable suggestions and feedback without whom our work would have been very difficult. We are highly indebted to our guide Ms. Dhanashree Toradmalle for her exemplary guidance, monitoring and constant encouragement throughout the course of this project. The blessing, help and guidance given by her time to time shall carry us a long way in the journey of life on which we are about to embark.

\section{References}

[1] E.-A. Baatarjav and R. Dantu, “Current and Future Trends in Social Media,” 2011 IEEE Third Int'l Conference on Privacy, Security, Risk and Trust and 2011 IEEE Third Int'l Conference on Social Computing, 2011.

[2] T.-M. Gronli, J. Hansen, G. Ghinea, and M. Younas, "Mobile Application Platform Heterogeneity: Android vs Windows Phone vs. iOS vs. Firefox OS,” 2014 IEEE 28th International Conference on Advanced Information Networking and Applications, 2014.

[3] S. Guo-Hong, “Application Development Research Based on Android Platform,” 2014 7th International Conference on Intelligent Computation Technology and Automation, 2014.

[4] A. G. Pepita and T. Juhana, "User Interface, Creation and Retrieval of User Health Information with Google Firebase, and Delivery of Automatic Emergency SMS for Ambient Assisted Living System: Monitoring of Elderly Condition Using Smart Devices,” 2018 4th International Conference on Wireless and Telematics (ICWT), 2018.

\section{Review of concepts and related works:}

Evaluation of paper [1]:

This paper helped us with our research objective of Public Attitude, and gave us the insight on how people like to interact on a daily basis and gave us the perspective to harness the power of Social Media.

Evaluation of paper [2]:

One of the major research objectives was to find the best platform, this paper provided the insight that maximum reach can be attained by having a mobile application, that to working both on android and iOS, only the e can have considerable impact.

Evaluation of paper [3]:

There is also a need for an application that can provide management solutions that need not be as sophisticated as the application as a user app, but shall require some complicated functionality, such as background tasks, location based 
services, fetching and computing background data. The paper provided us directions that for such tasks android shall be the most economical and feasible option.

Evaluation of paper [4]:

This paper helped us to get near to our objective of making the application affordable and have minimal maintenance cost, it provided us with the options of using Google firebase services which provided just necessary backend services at Zero Cost, also inspired us that how can we provide a better user experience to the user.

\section{Authors' Profiles}

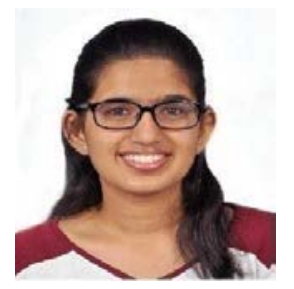

Ms. Janhavi Desale is currently studying Bachelor of Engineering in Information Technology from Shah and Anchor Kutchhi Engineering. Her research interests include Database Management, Ethical Hacking, User Interaction Design and Cyber Security. She is the Public Relations Officer of the Research Cell at SAKEC. She also reached the final level of Smart India Hackathon (software edition) and has received an honourable mention in All India Essay Writing Event in 2018.

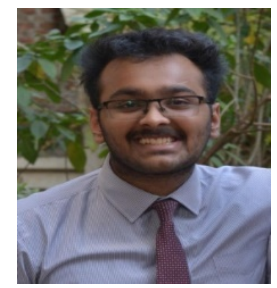

Mr. Kunal Gautam is currently pursuing Bachelor of Engineering in Information Technology from Shah and Anchor Kutchhi Engineering College. He can be referred to as someone with an unquenchable appetite for knowledge. He relishes researching in domains like Cyber Security, Artificial Intelligence. His keen interest in the field of Cyber Security has provided him with an opportunity to fly abroad for his Master's to MONASH University, Melbourne, Australia.

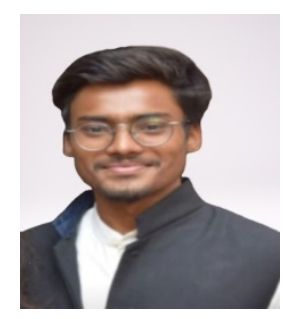

Mr. Saish Khandare is currently pursuing Bachelor of Engineering in Information Technology from Shah and Anchor Kutchhi Engineering College. His research interests include Software Engineering and Programming, Data Science, User Interaction Design, Cyber Security. Proficient in co-curricular activities he is the Vice President of the Research Cell at SAKEC.

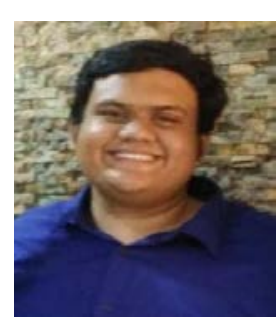

Mr. Vedant Parikh is currently pursuing Bachelor of Engineering in Information Technology from Shah and Anchor Kutchhi Engineering College (SAKEC), affiliated to Mumbai University. His research interests include Artificial Intelligence, DevOps, Ethical Hacking and Cyber Security. He is the Secretary of the Research Cell at SAKEC. He is also passionate about social service.

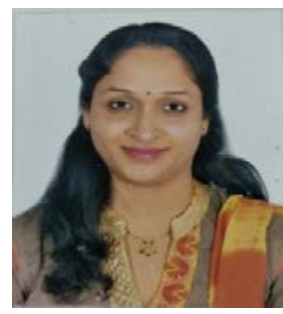

Ms. Dhanashree Toradmalle is an Associate Professor in Information Technology Department of Shah and Anchor Kutchhi Engineering College. Her areas of interests are Automation of administrative tasks for Teaching learning processes, Mobile and Web development, Information Security and Computer Networks.

How to cite this paper: Janhavi Desale, Kunal Gautama, Saish Khandare, Vedant Parikh, Dhanashree Toradmalle. " NGO Support Software Solution : for effective reachability ", International Journal of Education and Management Engineering (IJEME), Vol.10, No.6, pp.17-26, 2020. DOI: 10.5815/ijeme.2020.06.03 